\title{
Separation of Isomers L-Alanine and Sarcosine in Urine by Electrospray Ionization and Tandem Differential Mobility Analysis-Mass Spectrometry
}

\author{
Pablo Martínez-Lozano ${ }^{a}$ and Juan Rus ${ }^{b}$ \\ ${ }^{a}$ Institute for Biomedical Technologies-National Research Council, Milan, Italy \\ b SEADM, Valladolid, Spain
}

Sarcosine, an isomer of $L$-alanine, has been proposed as a prostate cancer progression biomarker [1]. Both compounds are detected in urine, where the measured sarcosine/alanine ratio has been found to be higher in prostate biopsy-positive group versus controls. We present here preliminary evidence showing that urine samples spiked with sarcosine/alanine can be partially resolved in $3 \mathrm{~min}$ via tandem differential mobility analysis-mass spectrometry (DMA-MS). Based on the calibration curves obtained for two mobility peaks, we finally estimate their concentration ratio in urine. (J Am Soc Mass Spectrom 2010, 21, 1129-1132) (C) 2010 American Society for Mass Spectrometry

$\mathrm{P}$ rostate cancer is a leading cause of death among the male population. Sarcosine, an isomer of alanine, was recently proposed as a prostate cancer progression biomarker [1]. In particular, the sarcosine/alanine ratio was found to be significantly higher in urine derived from biopsy-positive prostate cancer patients compared with biopsy-negative controls. This finding could be an important milestone for noninvasive diagnosis of prostate cancer. The technique used in [1] to assess sarcosine levels in urine is GC-MS. GC-MS is a time-consuming procedure, so the development of faster separation alternatives would be highly desirable if the method were to be applied in large-scale clinical practice. Ion mobility spectrometry-mass spectrometry (IMS-MS) can represent such an alternative.

Most IMS-MS work has relied on linear drift tubes. The majority of previous IMS-MS studies of biomolecules have dealt with proteins [2], with fewer studies targeting the metabolome [3] as we do here. Differential mobility analyzers (DMAs) separate species of different mobilities using space-dispersion rather than time-dispersion [4]. This feature makes them easier to combine with secondstage analyzers than traditional linear drift tubes. We explore here the ability of a DMA-MS system to discriminate the isomers $L$-alanine and sarcosine in urine.

\section{Experimental}

The coupling in series of a DMA (SEADM) to a commercial QTOF (SCIEX's QStar, Concord, ON, Canada) for mobility and mass measurement was described in detail elsewhere $[4,5]$. We electrosprayed our samples in posi-

Address reprint requests to Dr. P. Martínez-Lozano, Institute for Biomedical Technologies-National Research Council, Via Fratelli Cervi 93, 20090 Segrate, Milan, Italy. E-mail: pablo.mlsinues@gmail.com tive-ion mode at the DMA entrance slit. Our nanospray parameters were: capillary $360 \mu \mathrm{m}$ o.d., $50 \mu \mathrm{m}$ i.d., length $22 \mathrm{~cm}$, driving pressure $75 \mathrm{mbar}$, and $3.8 \mathrm{kV}$. The ions entered the separation region propelled by an electric field and against a counterflow gas $(0.2 \mathrm{~L} / \mathrm{min})$. Sheath gas flow was kept constant, and the classification voltage was scanned. Typical operation sheath flow rates were in the range of $1,000 \mathrm{~L} / \mathrm{min}$, and scanning voltages in the range of 1.0-2.6 kV. Mobility-selected ions entered the mass spectrometer to be analyzed according to their $\mathrm{m} / \mathrm{z}$.

We first analyzed sarcosine and $L$-alanine standards separately (100 $\mu \mathrm{M}$ in methanol, formic acid $2 \%$ ), and subsequently we analyzed them as a mixture $(50 \mu \mathrm{M}$ each in methanol, formic acid $2 \%$ ). Upon characterization of the standards we analyzed urine samples spiked with alanine or sarcosine. In this case, the spiked samples were diluted (1:9:0.3 urine:methanol:formic acid) and were electrosprayed without further sample preparation. Collision induced dissociation (CID) experiments were performed selecting a desired mobility by fixing the DMA voltage, and then using the QStar standard MS-MS methods, at a collision energy of $10 \mathrm{~V}$.

\section{Results and Discussion}

\section{Standards}

Figure 1 shows the mobility spectra of protonated $L$-alanine and sarcosine $(100 \mu \mathrm{M} ; m / z 90.06 \pm 0.01$ for both), with the tetraheptylammonium ion used as internal standard in both samples $\left(\mathrm{THA}^{+} 10 \mu \mathrm{M} ; \mathrm{m} / \mathrm{z}\right.$ $410.14 \pm 0.01$ ), in $\mathrm{N}_{2}$ as drift gas. Note that for both experiments the $\mathrm{THA}^{+}$peak appears overlapped, while the sarcosine and $L$-alanine profiles are partially displaced. Measured resolution for the $\mathrm{THA}^{+}$peak was 42 , 


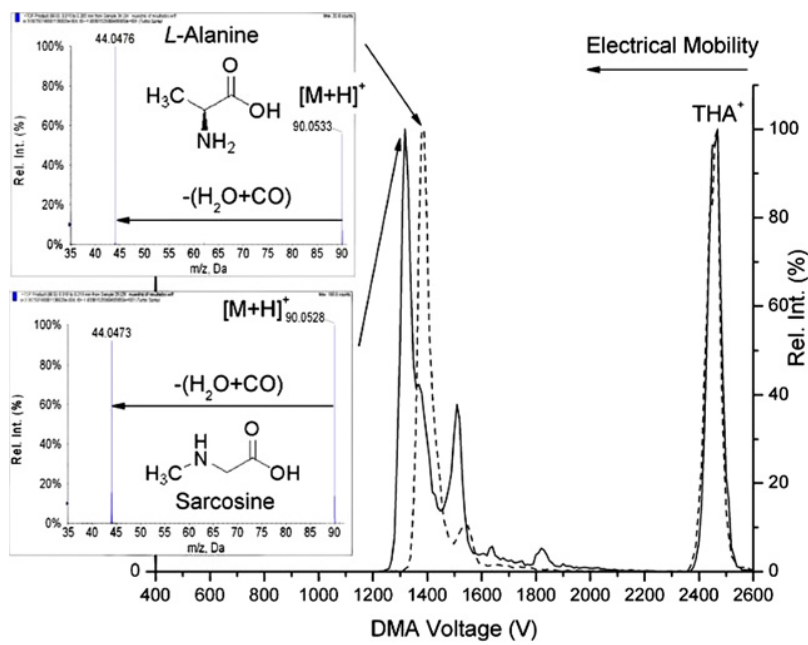

Figure 1. Mobility spectra in $\mathrm{N}_{2}$ for alanine, sarcosine and $\mathrm{THA}^{+}$ (the latter is spiked in both solutions). The insets display the MS/MS spectra from the dominant ion in the mobility spectrum.

lower than the usual values $(>50)$ for this DMA (this will be discussed below). The insets show the corresponding CID spectra of the most abundant ion in the ion-mobility spectrum, showing the same characteristic fragment at $\mathrm{m} / \mathrm{z} 44.05$ for both isomers. This is in excellent agreement with the database www.massbank.jp. Therefore, because there is no characteristic fragment that is isomer-specific, clearly MS/MS alone does not suffice to discriminate them in a mixture, and a previous separation stage is needed. The ion at $\mathrm{m} / \mathrm{z}$ 44.05 corresponds to the immonium ion derived by loss of $\mathrm{H}_{2} \mathrm{O}+\mathrm{CO}$ [6]. Note finally that in contrast with drift tubes, fragmentation studies of a mixture of isomers are done by simply selecting their mobility in the DMA, removing isomers of different mobilities before mass analysis.

In contrast with the $\mathrm{THA}^{+}$well-defined peak, the mobility spectra for sarcosine and alanine display multiple features apart from the main, high mobility peak. In an attempt to identify these mobility features, we also performed CID experiments on them, all of which showed the same fragmentation pattern. For this reason, we believe the lower mobility peaks are simply clusters of either sarcosine or alanine with solvent molecules that do not survive the transition between atmospheric pressure (DMA) and low pressure (MS). They also include contributions from dimers and trimers dissociating between the mobility and the mass analysis. We have monitored these $(\mathrm{m} / \mathrm{z} 179$ dimer and $\mathrm{m} / \mathrm{z} 268$ trimer) in another series of experiments (not shown), and they make a minor contribution to the $\mathrm{m} / \mathrm{z}$ 90 mobility spectrum, accounting for some small features in the long, low mobility tail.

Another advantageous feature of DMAs is that they operate in the linear regime (i.e., weak electric field). The electrical mobility of the $\mathrm{THA}^{+}$ion was previously characterized in air at $20^{\circ} \mathrm{C}$ [7], so we spiked the sarcosine/alanine solutions with this internal standard and ran the samples in air (Figure S1 Supplemental Material, which can be found in the electronic version of this article). We monitored the temperature at the wall at the DMA exit, which was $31^{\circ} \mathrm{C}$ for both alanine and sarcosine runs shown in Figure S1. With this measurement and an estimation of the heating due to the dynamic pressure wasted or isentropically recovered in the diffuser (efficiency of $\sim 50 \%$ ), we can approximately estimate a $26^{\circ} \mathrm{C}$ temperature in the classification region. Measured mobilities for sarcosine and alanine, corrected to $20^{\circ} \mathrm{C}$ as $K \sim \mathrm{T}^{1 / 2}$ in air at $1 \mathrm{~atm}$, were 1.94 and $1.89 \mathrm{~cm}^{2} \mathrm{~V}^{-1} \mathrm{~s}^{-1}$, respectively. It is interesting to note that even though these ions approach the mobility attributable to polarization forces alone, given by the polarization limit $\left(\sim 2.2 \mathrm{~cm}^{2} \mathrm{~V}^{-1} \mathrm{~s}^{-1}\right)$ [8], we still observe a size effect that enables their separation. This is in accordance with Johnson et al. [9], who measured a reduced mobility $\left(K_{0}\right)$ in $\mathrm{N}_{2}$ of 1.982 and $1.821 \mathrm{~cm}^{2} \mathrm{~V}^{-1} \mathrm{~s}^{-1}$ for sarcosine and $\beta$-alanine, respectively. These data indicate that IMS-MS may become an attractive analytical approach for metabolic profiling, even for the smallest target molecules.

It is well known that an appropriate selection of the drift gas results in an improvement of separability. As shown in Figure S1, the separation in air is less efficient than in $\mathrm{N}_{2}$, and we also tried the separation in $\mathrm{CO}_{2}$, again with worse separability than in $\mathrm{N}_{2}$. This is in agreement with the separation of sarcosine and $\beta$-alanine in [9]. Therefore, all subsequent data are for $\mathrm{N}_{2}$ as drift gas. Note finally that the mobility spectra in Figure 1 show less low-mobility features than those of Figure S1. We found that the temperature plays a critical role in the profile of the mobility spectra. Spectra from Figure 1 were recorded at $27-28^{\circ} \mathrm{C}$ at the DMA exit (it was $31^{\circ} \mathrm{C}$ in Figure S1), and they show a dominating high mobility peak and much weaker low mobility peaks, whereas at $45{ }^{\circ} \mathrm{C}$ we observed an unresolved broad peak containing several mobilities. Because the main peak of interest here is the one of highest mobility, which most likely corresponds to the bare ion, we restricted DMA operation below $32^{\circ} \mathrm{C}$.

Therefore, we conclude from these preliminary experiments that the mobility of sarcosine (based on the highest mobility peak) in $\mathrm{N}_{2}$ is $\sim 4.5 \%$ higher than that of $L$-alanine.

Figure S2 shows the mobility spectra recorded at increasing sheath gas flow rate (i.e., increasing resolution) of an equimolar mixture $(50 \mu \mathrm{M})$ of both isomers. One can observe how, at increasing resolution, the main broad peak tends to split into two peaks corresponding to sarcosine and alanine. Note finally that during these series of experiments, we lacked an extension of the DMA diffuser that provides additional dynamic pressure recovery. As a result, the system tended to heat over $35^{\circ} \mathrm{C}$ even at moderate gas speeds. For this reason, we were forced to operate at slightly lower resolutions than maximum capacity. 


\section{Urine}

Once we assessed the capabilities of the system to separate both standards, we tested its response to real urine samples. Figure 2a displays the resulting mobility spectrum $(\mathrm{m} / \mathrm{z}$ 90.05-90.07) of a diluted urine sample (bottom pane). The same sample was spiked with sarcosine or alanine at increasing concentrations (range 24-87 $\mu \mathrm{M}$ ). Three main features around 1.24 (A1), 1.35 (A2), and 1.45 (A3) kV consistently increased at increas- ing alanine concentration. Spiking with sarcosine clearly increases a shoulder at $\sim 1.20$ (S1) kV over A1 and two more features at 1.30 (S2) and 1.44 (S3) kV. To provide further evidence of the identity of the peaks, we performed MS/MS experiments. The insets show the CID spectra obtained by fixing the DMA voltage to select the highest mobility ion (S1 or A1) for the most concentrated sarcosine and alanine-spiked samples. Both product-ion spectra show the characteristic frag-
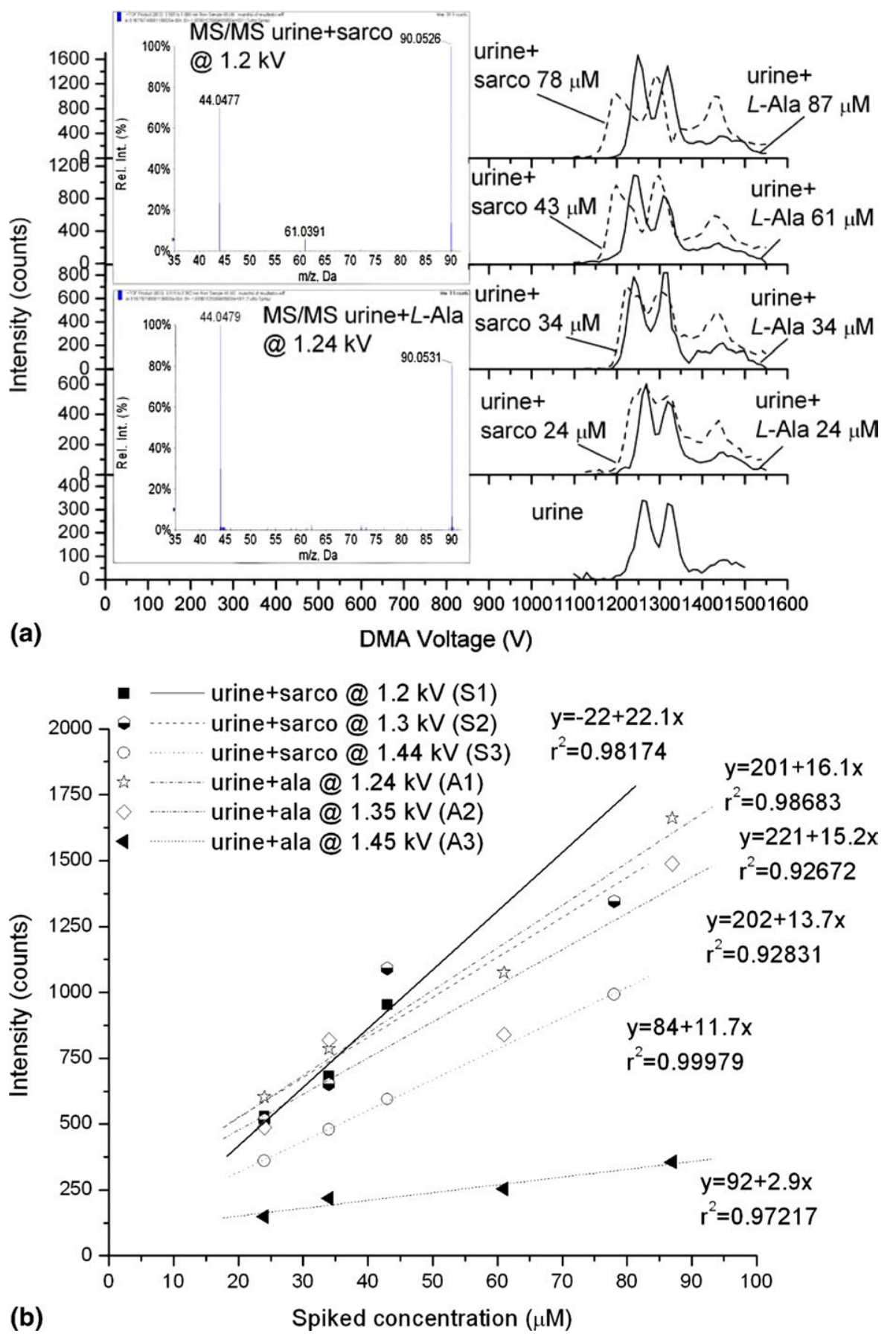

Figure 2. (a) Top: mobility spectra for a urine sample, unspiked and spiked with either alanine or sarcosine at increasing concentrations. (b) Bottom: calibration curves obtained for the main three features associated to both isomers in (a). 
mentation pattern of alanine and sarcosine, although the latter also shows a minor peak at $m / z 61$ from an unknown interference. Ions corresponding to the peaks labeled as 2 and 3 showed the same CID pattern as that for 1 , indicating that they are sarcosine/alanine clusters, although they remain to be fully characterized.

Figure $2 \mathrm{~b}$ collects the maximum intensity of the peaks considered as a function of the spiked concentration. It suggests a reasonable linearity for most of them, with some scattered data. Note that each sample was only run once and that we have discarded the most concentrated S1 peak because, for unclear reasons, it falls completely off the main trend. It also shows a similar sensitivity $(\sim 10-20$ counts $/ \mu \mathrm{M})$ for all of them except for the weak $1.45 \mathrm{kV}$ alanine peak. This peak is nevertheless unresolvable by this DMA from the 1.40 $\mathrm{kV}$ sarcosine peak.

Peak deconvolution is a useful approach to resolve incompletely separated peaks. Figure 3 shows the original and deconvoluted spectra (Supporting Information) from Figure $2 a$, for the unspiked urine and the 34 $\mu \mathrm{M}$ spiked sarcosine samples. Area ratios between the main peaks are $\sim 0.36$ in the unspiked sample (both S1/A1 and S2/A2) and 1.1 in the spiked sample (again for both of them). Considering the sensitivities estimated in Figure $2 b$ for each of the peaks, this results in sarcosine/alanine ratios of $\sim 0.3$ and 0.9 , respectively. Figure 3, therefore, illustrates the expected typical mobility profile from a control (bottom) and a prostate cancer patient (top). This quantification also implies a $\sim 60 \mu \mathrm{M}$ alanine concentration in the $1 / 10$ diluted urine sample, which is also roughly consistent (within order of magnitude) with reported concentrations of alanine in urine $(\sim 270 \mu \mathrm{M})[10]$, indicating a minimal ion suppression effect in this particular case.

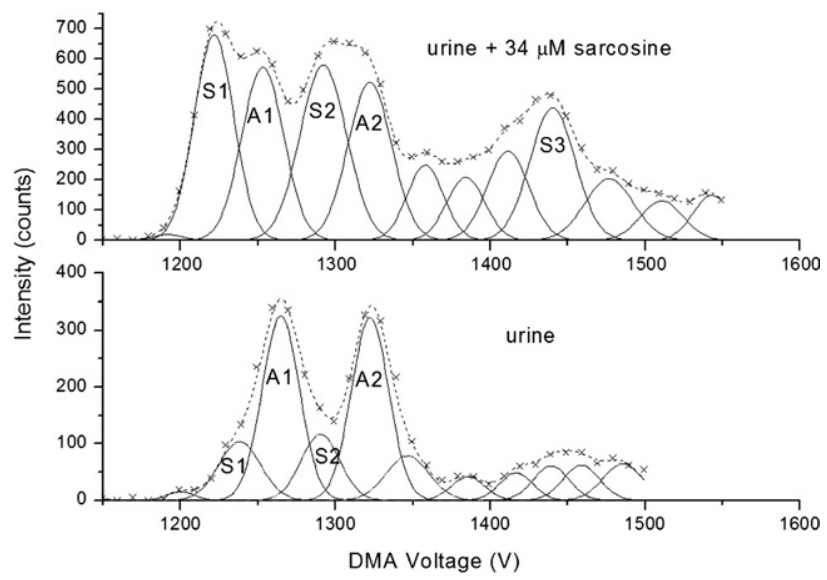

Figure 3. Deconvoluted mobility spectra for the unspiked urine (bottom) and the same urine spiked with sarcosine (top). S stands for sarcosine and A for alanine. Inferred sarcosine/alanine ratios for top and bottom figures are 0.9 and 0.3 , respectively.

\section{Conclusions}

We separated sarcosine/alanine mixtures with an ESIDMA-MS system. Preliminary experiments with pure standards indicated that electrical mobility of sarcosine is $\sim 4.5 \%$ higher than that of $L$-Alanine in nitrogen as drift gas. Experiments on spiked urine revealed that both isomers can also be partially resolved with minimal sample preparation (analysis time $\sim 3 \mathrm{~min}$ ). Deconvolution of unresolved sarcosine and alanine peaks enables their concentration ratio estimation.

\section{Acknowledgments}

P.M.L. thanks SEADM for providing access to their DMA-QTOF system. Ms. M. Hernández, Mr. A. Casado, Mr. D. Moro, Mr. G. Vidal, and rest of the SEADM team are gratefully acknowledged for their assistance during the experiments. The authors are most grateful to Professor Juan Fernández de la Mora for his guidance in the art of differential mobility analysis. The research leading to these results has received funding from the European Community's Seventh Framework Programme FP7/2007-2013 under grant agreement PIEF-GA-2008-220511.

\section{Appendix A Supplementary Material}

Supplementary material associated with this article may be found in the online version at doi:10.1016/ j.jasms.2010.02.021.

\section{References}

1. Sreekumar, A.; Poisson, L. M.; Rajendiran, T. M.; Khan, A. P.; Cao, Q.; Yu, J.; Laxman, B.; Mehra, R.; Lonigro, R. J.; Li, Y.; Nyati, M. K.; Ahsan, A.; Kalyana-Sundaram, S.; Han, B.; Cao, X.; Byun, J.; Omenn, G. S.; Ghosh, D.; Pennathur, S.; Alexander, D. C.; Berger, A.; Shuster, J. R.; Wei, J. T.; Varambally, S.; Beecher, C.; Chinnaiyan, A. M. Metabolomic Profiles Delineate Potential Role for Sarcosine in Prostate Cancer Progression. Nature 2009, 457(7231), 910-914.

2. Bernstein, S. L.; Liu, D. F.; Wyttenbach, T.; Bowers, M. T.; Lee, J. C.; Gray, H. B.; Winkler, J. R. $\alpha$-Synuclein: Stable Compact and Extended Monomeric Structures and pH Dependence of Dimer Formation. J. Am. Soc. Mass Spectrom. 2004, 15, 1435-1443.

3. Kaplan, K; Dwivedi, P; Davidson, S; Yang, Q; Tso, P; Siems, W; Hill, H. H. Monitoring Dynamic Changes in Lymph Metabolome of Fasting and Fed Rats by Electrospray Ionization-Ion Mobility Mass Spectrometry (ESI-IMMS). Anal. Chem. 2009, 81, 7944-7953.

4. Rus, J.; Moro D.; Sillero J. A.; Royuela, J.; Casado, A.; Estévez-Molinero, F.; Fernández de la Mora, J. IMS-MS Studies Based on Coupling a Differential Mobility Analyzer (DMA) to Commercial API-MS Systems, unpublished (submitted).

5. Hogan C. J.; Fernández de la Mora J. Tandem Ion Mobility-Mass Spectrometry (IMS-MS) Study of Ion Evaporation from Ionic LiquidAcetonitrile Nanodrops. Phys. Chem., Chem. Phys. 2009, 11, 8079-8090.

6. Dookeran, N. N.; Yalcin, T.; Harrison, A. G. Fragmentation Reactions of Protonated $\alpha$-Amino Acids. J. Mass Spectrom. 1996, 31, 500-508.

7. Gamero-Castaño, M.; Fernández de la Mora, J. Mechanisms of Electrospray Ionization of Singly and Multiply Charged Salt Clusters. Anal. Chim. Acta 2000, 406, 67-91.

8. Mason, E. A.; McDaniel, E. W. Transport Properties of Ions in Gases; Wiley: New York, 1988; p. 245.

9. Johnson, P. V.; Kim, H. I.; Beegle L. W.; Kanik I. Electrospray Ionization Ion Mobility Spectrometry of Amino Acids: Ion Mobilities and a Mass-Mobility Correlation. J. Phys. Chem. A 2004, 108(27), 5785-5792.

10. Shaykhutdinov, R. A.; MacInnis, G. D.; Dowlatabadi, R.; Weljie, A. M.; Vogel, H. J. Quantitative Analysis of Metabolite Concentrations in Human Urine Samples Using ${ }^{13} \mathrm{C}\left\{{ }^{1} \mathrm{H}\right\}$ NMR Spectroscopy. Metabolomics 2009, 5, 307-317. 